\title{
A NOETHER-LEFSCHETZ THEOREM FOR VARIETIES OF $r$-PLANES IN COMPLETE INTERSECTIONS
}

\author{
ZHI JIANG
}

\begin{abstract}
We prove a Noether-Lefschetz type theorem for varieties of $r$-planes in complete intersections. We then use it to study the Abel-Jacobi map of planes on a smooth cubic fivefold.
\end{abstract}

\section{$\S 0$. Introduction}

Let $X$ be a general complete intersection in complex projective space. The Picard number of $X$ is known. We may state it in the following form.

TheOREM 0.1. Let $X$ be a smooth complete intersection of dimension at least 2 in complex projective space.

- If $\operatorname{dim} X \geq 3$, the second Betti number of $X$ is 1 , and in particular, the Picard number $\rho(X)$ of $X$ is 1 .

- If $\operatorname{dim} X=2$ and if $X$ is very general, the Picard number $\rho(X)$ is 1 , except when $X$ is a quadric surface in $\mathbf{P}^{3}$, or $X$ is a cubic surface in $\mathbf{P}^{3}$, or $X$ is a complete intersection of two quadrics in $\mathbf{P}^{4}$.

The first part of the above theorem comes from the Lefschetz hyperplane theorem and the second part is the so-called Noether-Lefschetz theorem (see [V1, Section 3] or [S, Section 2]). Bonavero and Voisin [BV], using Deligne's global invariant cycles theorem, proved an analogue of the first part of the above theorem for the schemes parameterizing $r$-planes contained in a complete intersection in complex projective space. Debarre and Manivel $[\mathrm{DM}]$ later used Bott's theorem to give another proof of the same theorem. The goal of this paper is to complete their result. We will prove an analogue of the second part of Theorem 0.1 for such schemes.

We first recall some notation.

We follow the presentation in $[\mathrm{DM}]$. Let $V$ be a complex vector space of dimension $n+1$. For a finite sequence $\underline{d}=\left(d_{1}, \ldots, d_{s}\right)$ of integers at least 2

Received January 26, 2011. Revised August 24, 2011. Accepted August 24, 2011.

2010 Mathematics Subject Classification. Primary 14C22, 14D07; Secondary 14D05.

(C) 2012 by The Editorial Board of the Nagoya Mathematical Journal 
and a positive integer $r$, we set $|\underline{d}|=\sum_{i=1}^{s} d_{i}, \underline{d}+r=\left(d_{1}+r, \ldots, d_{s}+r\right)$, and $d / r=\sum_{i=1}^{s}\left(\begin{array}{c}d_{i} \\ r\end{array}\right)$.

Let $X \subset \mathbf{P}(V)$ be a complete intersection defined by $f_{1}=\cdots=f_{s}=0$, where $f_{i} \in \mathbf{S}^{d_{i}} V^{*}$ for each $1 \leq i \leq s$. We denote by $F_{r}(X)$ the subscheme of $G:=G(r+1, V)$ parameterizing linear spaces of dimension $r$ contained in $X$. On $G(r+1, V)$, there is the tautological subbundle $\Sigma$ of $V \otimes \mathscr{O}_{G}$ of rank $r+1$ and the tautological quotient bundle $Q$ of rank $n-r$, and $\Omega_{G}^{1} \simeq \Sigma \otimes Q^{*}$. Each $f_{i}$ induces a global section $\sigma_{i}$ of $\mathbf{S}^{d_{i}} \Sigma^{*}$ on $G$. We can also see $F_{r}(X)$ as the zero locus of the global sections $\sigma_{i}$ for all $1 \leq i \leq s$. We then set

$$
\delta(n, \underline{d}, r)=(r+1)(n-r)-\left(\begin{array}{c}
\underline{d}+r \\
r
\end{array}\right),
$$

which is the expected dimension of $F_{r}(X)$, and set

$$
\delta_{-}(n, \underline{d}, r)=\min \{\delta(n, \underline{d}, r), n-2 r-s\} .
$$

There are many works about the schemes $F_{r}(X)$. The basic properties of $F_{r}(X)$ are studied in $[\mathrm{AK}],[\mathrm{B} 1],[\mathrm{BVV}]$, and [DM]. As interesting examples, the schemes $F_{r}(X)$ have been considered in different context (see [B2], [BD], $[\mathrm{BV}]$, and $[\mathrm{J}]$ ), and there are important applications to $X$ (see $[\mathrm{BM}],[\mathrm{CG}]$, and $[\mathrm{ELV}])$.

The following theorem is our starting point.

Theorem 0.2. Assume that $X$ is a general complete intersection as above.

(1) If $\delta_{-}(n, \underline{d}, r) \geq 1$, the scheme $F_{r}(X)$ is connected, smooth, and of dimension $\delta(n, \underline{d}, r)$ (see [B1] and [DM]).

(2) If $\delta_{-}(n, \underline{d}, r) \geq 3$, the second Betti number of $F_{r}(X)$ is 1 , and in particular, the Picard number $\rho\left(F_{r}(X)\right)$ of $F_{r}(X)$ is 1 (see [BV] and [DM]).

The above theorem is optimal. Indeed, when $\delta_{-}(n, \underline{d}, r) \leq 2$, the Hodge number $h^{2,0}\left(F_{r}(X)\right)$ is often nonzero. Hence, though we know the second Betti number of $F_{r}(X)$, it is a priori not clear what the Picard number is.

The main theorem of this paper is the following.

THEOREM 0.3. Let $X$ be a very general complete intersection in projective space. Assume that $\delta(n, \underline{d}, r) \geq 2$ and that $\delta_{-}(n, \underline{d}, r)>0 .^{\dagger}$ We have $\rho\left(F_{r}(X)\right)=1$ except in the following cases:

\footnotetext{
${ }^{\dagger}$ This assumption is not important. We use it only to exclude the case when $X$ is a quadric in $\mathbf{P}^{2 r+1}$ for $r \geq 1$, where $F_{r}(X)$ has two smooth isomorphic disjoint components and the Picard number of each component is 1 .
} 
- $X$ is a quadric in $\mathbf{P}^{2 r+3}, r \geq 1$, where the Picard number of $F_{r}(X)$ is 2;

- $X$ is a complete intersection of two quadrics in $\mathbf{P}^{2 r+4}, r \geq 1$, where the Picard number of $F_{r}(X)$ is $2 r+6$.

In the following examples, we will see that when $\delta_{-}(n, \underline{d}, r) \leq 2$, the Picard number $\rho\left(F_{r}(X)\right)$ varies, where the situation is similar to the families of smooth hypersurfaces of degree at least 4 in $\mathbf{P}^{3}$.

EXAmple 0.4. Let $X \hookrightarrow \mathbf{P}^{2 r+3}$ be a general complete intersection of two quadrics, where $r \geq 1$. We have $\delta(2 r+3, \underline{d}, r)=r+1$ and $\delta_{-}(2 r+3, \underline{d}, r)=1$. Hence, $F_{r}(X)$ is a smooth irreducible projective variety of dimension $r+1$.

We may assume that the two quadrics defining $X$ are

$$
\sum_{i} x_{i}^{2} \quad \text { and } \quad \sum_{i} \lambda_{i} x_{i}^{2}
$$

where $\lambda_{i} \neq \lambda_{j}$ if $i \neq j$. By [Re], we know that $F_{r}(X)$ is an abelian variety and is isomorphic to the Jacobian of the hyperelliptic curve defined by

$$
y^{2}=\Pi_{i}\left(x_{0}-\lambda_{i} x_{1}\right) .
$$

Therefore, by $[\mathrm{P}], \rho\left(F_{r}(X)\right)=1$ for a very general $X$. There also exist smooth $X$ such that $\rho\left(F_{r}(X)\right) \geq 2$, for instance, when the hyperelliptic curve is defined by $y^{2}=x^{2 r+4}-1$.

EXAmple 0.5 (see [CG]). Let $X \hookrightarrow \mathbf{P}^{4}$ be a cubic threefold. We consider $F_{1}(X)$ and have in this case $\delta(4,3,1)=2$ and $\delta_{-}(4,3,1)=1$.

Clemens and Griffiths [CG] proved that the Abel-Jacobi map $\alpha: F_{1}(X) \rightarrow$ $J X$ induces an isomorphism $\alpha_{*}: \operatorname{Alb}\left(F_{1}(X)\right) \simeq J X$ and $\wedge^{2} H^{1}\left(F_{1}(X), \mathbf{Q}\right) \simeq$ $H^{2}\left(F_{1}(X), \mathbf{Q}\right)$. We conclude that

$$
H^{2}\left(F_{1}(X), \mathbf{Q}\right) \simeq H^{2}(J X, \mathbf{Q}) \simeq \wedge^{2} H^{3}(X, \mathbf{Q}) .
$$

Since the Zariski closure of the monodromy group for the family of cubic threefolds is the whole symplectic group (see [PS, Theorem 10.22]), we have $\rho\left(F_{1}(X)\right)=1$ for $X$ very general. We see in [Ro] that there is a 7-dimensional family in the 10-dimensional moduli space of cubic threefolds parameterizing cubics $X$ whose variety of lines $F_{1}(X)$ contains elliptic curves. For such $X$, we have $\rho\left(F_{1}(X)\right) \geq 2$.

REMARK 0.6. Roulleau showed me a more precise relation between the Néron-Severi group of $F_{1}(X)$ and $J X$ :

$$
0 \rightarrow \mathrm{NS}(J X) \stackrel{\alpha^{*}}{\longrightarrow} \mathrm{NS}\left(F_{1}(X)\right) \rightarrow \mathbf{Z} / 2 \mathbf{Z} \rightarrow 0 .
$$


Example 0.7. Let $X \hookrightarrow \mathbf{P}^{7}$ be a cubic sixfold. We have $\delta(7,3,2)=5$ and $\delta_{-}(7,3,2)=2$.

The 2-planes cover the whole of $X$; hence, the Abel-Jacobi map induces an injective map $H^{6}(X, \mathbf{Q})_{\text {prim }} \rightarrow H^{2}\left(F_{2}(X), \mathbf{Q}\right)$. We can also compute that

$$
\operatorname{dim} H^{6}(X, \mathbf{Q})=\operatorname{dim} H^{2}\left(F_{2}(X), \mathbf{Q}\right)=87 .
$$

Hence, since the monodromy group of the family of cubic of dimension 6 is again big [PS, Theorem 10.22], if $X$ is general, $\rho\left(F_{2}(X)\right)=\operatorname{rank}\left(H^{3,3}(X) \cap\right.$ $\left.H^{6}(X, \mathbf{Q})\right)=1$. If $X$ contains some special codimension 3 subvariety (e.g., $\left.\mathbf{P}^{3}\right)$, we have $\rho\left(F_{2}(X)\right)=\operatorname{rank}\left(H^{3,3}(X) \cap H^{6}(X, \mathbf{Q})\right) \geq 2$.

We will give in the last section the following application of our NoetherLefschetz-type theorem.

For any smooth cubic fivefold $Z$, we denote by $(J Z, \Theta)$ the principally polarized intermediate Jacobian of $Z$ which is a 21-dimensional principally polarized abelian variety. We then have the Abel-Jacobi map

$$
\alpha: F_{2}(Z) \rightarrow J Z
$$

As an application of our main theorem, we have the following.

THEOREM 0.8. With the notation as above, the cohomology class

$$
\left[\alpha_{*}\left(F_{2}(Z)\right)\right]=12\left[\frac{\Theta^{19}}{19 !}\right] .
$$

\section{$\S 1$. Preliminaries}

We will use deformation arguments to prove the main theorem. In this section, we first recall a lemma which reduces the proof to certain calculations of cohomology groups, and then we recall Bott's theorem, which helps us to calculate the cohomology groups of homogeneous vector bundles on Grassmannians.

Let $Y$ be a smooth projective variety of dimension $N$ and Picard number 1 . Let $W$ be a vector bundle of rank $R$ on $Y$ with $N-R \geq 2$. For a section $\sigma$ of $W$, we denote by $X_{\sigma}$ the zero locus of $s$.

Lemma 1.1. We fix a general section $\sigma$ of $W$. Assume that $X_{\sigma}$ is smooth and of dimension $N-R$, and assume that we have

(a) $\operatorname{dim} H^{1}\left(X_{\sigma},\left.\Omega_{Y}^{1}\right|_{X_{\sigma}}\right)=1$; 
(b) $\left.H^{N-R-2}\left(X_{\sigma}, K_{X_{\sigma}}\right) \otimes H^{0}(Y, W)\right|_{X_{\sigma}} \rightarrow H^{N-R-2}\left(X_{\sigma}, W \otimes K_{X_{\sigma}}\right)$ is surjective.

Then for a very general section $\rho \in H^{0}(Y, W)$, the Picard number of the zero locus $X_{\rho}$ is 1 .

Proof. Let $U \subset H^{0}(Y, W)$ be the open subset parameterizing sections whose zero locus is smooth of the expected dimension. We set $\pi: \mathscr{X} \rightarrow U$ as the family of $X_{\sigma}$. We have the sequence

$$
\left.0 \rightarrow T_{X_{\sigma}} \rightarrow T_{\mathscr{X}}\right|_{X_{\sigma}} \rightarrow T_{U, \sigma} \rightarrow 0
$$

And for any $t \in T_{U, \sigma}$, we have the Kodaira-Spencer class $\delta(t) \in H^{1}\left(X_{\sigma}, T_{X_{\sigma}}\right)$, where $\delta$ is the coboundary map in (1).

We denote by $h$ the cohomology class of an ample divisor of $Y$, and we denote by $H^{1,1}\left(X_{\sigma}\right)_{\text {prim }}$ the primitive $(1,1)$-forms on $X_{\sigma}$ relative to $h$. In order to prove the lemma, we just need to show that for any $\lambda \in$ $H^{1,1}\left(X_{\sigma}\right)_{\text {prim }}$, the map

$$
\nabla_{\lambda} \circ \delta: H^{0}(Y, W) \simeq T_{U, \sigma} \rightarrow H^{2}\left(X_{\sigma}, \mathscr{O}_{X_{\sigma}}\right)
$$

is nontrivial (see, e.g., [V1, Chapter 5]).

Considering the sequence

$$
\left.0 \rightarrow W^{*} \rightarrow \Omega_{Y}^{1}\right|_{X_{\sigma}} \rightarrow \Omega_{X_{\sigma}}^{1} \rightarrow 0
$$

we have

$$
H^{1}\left(X_{\sigma},\left.\Omega_{Y}^{1}\right|_{X_{\sigma}}\right) \rightarrow H^{1}\left(X_{\sigma}, \Omega_{X_{\sigma}}^{1}\right) \stackrel{\delta}{\rightarrow} H^{2}\left(X_{\sigma}, W^{*}\right) .
$$

Since $\operatorname{dim} H^{1}\left(X_{\sigma},\left.\Omega_{Y}^{1}\right|_{X_{\sigma}}\right)=1$, we have that the map

$$
\delta: H^{1,1}\left(X_{\sigma}\right)_{\operatorname{prim}} \rightarrow H^{2}\left(X_{\sigma}, W^{*}\right)
$$

is injective. Thus, by Serre duality of the map (1), we just need to show that the map

$$
\left.H^{N-R-2}\left(X_{\sigma}, K_{X_{\sigma}}\right) \otimes H^{0}(Y, W)\right|_{X_{\sigma}} \rightarrow H^{N-R-2}\left(X_{\sigma}, W \otimes K_{X_{\sigma}}\right)
$$

is surjective to conclude that the Picard number of a general zero locus $X_{\rho}$ is 1 . 
In our situation, the ambient variety $Y$ will be the Grassmannian $G(r+$ $1, V)$, and the vector bundle $W$ will be $\bigoplus_{i=1}^{s} \mathbf{S}^{d_{i}} \Sigma^{*}$. Hence, in order to verify conditions (a) and (b) in Lemma 1.1, we need to study the cohomology groups of homogeneous vector bundles on Grassmannians. We first recall Bott's theorem. Since we work only on $G(r+1, V)$ in this paper, we will present Bott's theorem in an elementary way (see, e.g., [S, Section 4.6]).

We call a finite decreasing sequence of integers $c=\left(c_{1}, \ldots, c_{k}\right)$ a partition. For a vector space $W$ of dimension $k$, we denote by $\Gamma^{c} W$ the irreducible $\mathrm{GL}(W)$-module associated to $c$. For example, we have

$$
\begin{aligned}
\Gamma^{(k, 0, \ldots, 0)} W & =\mathbf{S}^{k} W, \\
\Gamma^{(0,0, \ldots,-k)} W & =\mathbf{S}^{k} W^{*}, \\
\Gamma^{(1,1, \ldots, 1)} W & =\operatorname{det} W .
\end{aligned}
$$

Let $b=\left(b_{1}, b_{2}, \ldots, b_{n-r}\right)$ and $a=\left(a_{1}, a_{2}, \ldots, a_{r+1}\right)$ be partitions. We define

$$
\begin{aligned}
\phi(b, a) & =\left(b_{1}, b_{2}, \ldots, b_{n-r}, a_{1}, a_{2}, \ldots, a_{r+1}\right)-(1,2, \ldots, n+1) \\
& =\left(c_{1}, c_{2}, \ldots, c_{n+1}\right)
\end{aligned}
$$

and we define the set $\Phi_{b, a}$ to be the set consisting of the pairs $(s, t)$ with $s<t$ and $c_{s}<c_{t}$. We denote by $i(b, a)$ the cardinality of $\Phi_{b, a}$. We then reorder $\phi(b, a)$ by making it decreasing, and we denote the resulting sequence by $\phi(b, a)^{+}$and set

$$
\psi(b, a):=\phi(b, a)^{+}+(1,2, \ldots, n+1) .
$$

If $\psi(b, a)$ is not decreasing, we have $\Gamma^{\psi(b, a)} V=0$ by convention. The following is a special case of Bott's theorem.

TheOREM 1.2 ([Bot, Theorem 1]). With the above notation, we have

(1) $H^{q}\left(G, \Gamma^{b} Q \otimes \Gamma^{a} \Sigma\right)=0$ if $q \neq i(b, a)$;

(2) $H^{i(b, a)}\left(G, \Gamma^{b} Q \otimes \Gamma^{a} \Sigma\right)=\Gamma^{\psi(b, a)} V$.

Here is a useful corollary.

Corollary 1.3. On the Grassmannian $G=G(r+1, V)$, assume that for some partition a, we have

$$
H^{q}\left(G, \Gamma^{a} \Sigma\right) \neq 0
$$

Then there exists $k \geq 0$ such that $q=k(n-r)$. 
We will often identify the partitions $\left(a_{1}, \ldots, a_{n-r}\right)$ and $\left(a_{1}, \ldots, a_{n-r}, 0\right)$. The following proposition is crucial.

Proposition 1.4. We keep the notation as above.

(1) Let $a_{1}$ and $a_{2}$ be partitions at least zero; then the multiplication map

$$
H^{0}\left(G, \Gamma^{a_{1}} \Sigma^{*}\right) \otimes H^{0}\left(G, \Gamma^{a_{2}} \Sigma^{*}\right) \rightarrow H^{0}\left(G, \Gamma^{a_{1}} \Sigma^{*} \otimes \Gamma^{a_{2}} \Sigma^{*}\right)
$$

is surjective.

(2) For any positive partition $a>0$ and some positive integer $d>0$, if there exists some $k \geq 1$ such that the multiplication

$$
H^{k(n-r)}\left(G, \Gamma^{a} \Sigma\right) \otimes H^{0}\left(G, \mathbf{S}^{d} \Sigma^{*}\right) \rightarrow H^{k(n-r)}\left(G, \Gamma^{a} \Sigma \otimes \mathbf{S}^{d} \Sigma^{*}\right)
$$

is not surjective, we have $a_{k} \geq n-r+k$ and $a_{k+1} \geq k+1$.

Proof. We first prove (1). By Theorem 1.2, we have $H^{0}\left(G, \Gamma^{a_{1}} \Sigma^{*}\right)=$ $\Gamma^{a_{1}} V^{*}$ and $H^{0}\left(G, \Gamma^{a_{2}} \Sigma^{*}\right)=\Gamma^{a_{2}} V^{*}$. We have by the Littlewood-Richardson rule that

$$
\begin{aligned}
& \Gamma^{a_{1}} \Sigma^{*} \otimes \Gamma^{a_{2}} \Sigma^{*}=\bigoplus_{v} N_{v} \cdot \Gamma^{v} \Sigma^{*} \\
& \Gamma^{a_{1}} V^{*} \otimes \Gamma^{a_{2}} V^{*}=\bigoplus_{v} N_{v}^{\prime} \cdot \Gamma^{v} V^{*} .
\end{aligned}
$$

Since $\operatorname{dim} V^{*}>\operatorname{rank} \Sigma^{*}, N_{v}^{\prime} \geq N_{v}$ for any partition $v$. Then we conclude the proof of (1) by Theorem 1.2.

For (2), we write

$$
\Gamma^{a} \Sigma \otimes \mathbf{S}^{d} \Sigma^{*}=\bigoplus_{\alpha} \Gamma^{\alpha} \Sigma,
$$

where by the Littlewood-Richardson rule, $\alpha$ goes through all partitions satisfying

$$
a_{1} \geq \alpha_{1} \geq a_{2} \geq \alpha_{2} \geq \cdots \geq a_{r+1} \geq \alpha_{r+1},
$$

and

$$
\sum_{i=1}^{r+1} \alpha_{i}=\sum_{i=1}^{r+1} a_{i}-d .
$$

Fix any such partition $\alpha$. 
If $a_{k}<n-r+k$, we have $\alpha_{k}<n-r+k$. Hence, by Bott's theorem, we have

$$
H^{k(n-r)}\left(G, \Gamma^{a} \Sigma\right)=H^{k(n-r)}\left(G, \Gamma^{a} \Sigma \otimes \mathbf{S}^{d} \Sigma^{*}\right)=0,
$$

which contradicts our hypothesis.

If $a_{k+1} \leq k$, we have $H^{k(n-r)}\left(G, \Gamma^{a} \Sigma\right) \simeq \Gamma^{\psi(0, a)} V \neq 0$. We now study the map

$$
\begin{aligned}
m_{\alpha}: H^{k(n-r)}\left(G, \Gamma^{a} \Sigma\right) \otimes H^{0}\left(G, \mathbf{S}^{d} \Sigma^{*}\right) & \rightarrow H^{k(n-r)}\left(G, \Gamma^{a} \Sigma \otimes \mathbf{S}^{d} \Sigma^{*}\right) \\
& \rightarrow H^{k(n-r)}\left(G, \Gamma^{\alpha} \Sigma\right) .
\end{aligned}
$$

Now we denote by $V_{1}$ a linear subspace of codimension $(r+1-k)$ of $V$, and we take a linear subspace $V_{2}$ of $V$ so that $V \simeq V_{1} \oplus V_{2}$. Let $G_{1}:=G\left(k, V_{1}\right)$ be the Grassmannian, and denote by $i: G_{1} \rightarrow G$ the natural embedding which sends a subspace $\Sigma_{1}$ of $V_{1}$ to a subspace $\Sigma_{1} \oplus V_{2}$ of $V$. Then we denote by $\bar{a}$ (resp., $\bar{\alpha})$ the partition $\left(a_{1}, \ldots, a_{k}\right)$ (resp., $\left.\left(\alpha_{1}, \ldots, \alpha_{k}\right)\right)$ and denote by $\widetilde{a}$ (resp., $\widetilde{\alpha})$ the partition $\left(a_{k+1}, \ldots, a_{r+1}\right)$ (resp., $\left.\left(\alpha_{k+1}, \ldots, \alpha_{r+1}\right)\right)$. Hence, $a=(\bar{a}, \widetilde{a})$ and $\alpha=(\bar{\alpha}, \widetilde{\alpha})$. Finally, we denote $d_{1}=\sum_{i=1}^{k}\left(a_{i}-\alpha_{i}\right)$ and $d_{2}=$ $\sum_{i=k+1}^{r+1}\left(a_{i}-\alpha_{i}\right)$. We have that both $d_{1}$ and $d_{2}$ are at least zero and that $d_{1}+d_{2}=d$. We notice that $\Gamma^{\bar{a}} \Sigma_{1} \otimes \Gamma^{\widetilde{a}} V_{2}$ (resp., $\mathbf{S}^{d_{1}} \Sigma_{1}^{*} \otimes \mathbf{S}^{d_{2}} V_{2}^{*}$ ) is a direct summand of $i^{*} \Gamma^{a} \Sigma$ (resp., $\left.i^{*} \mathbf{S}^{d} \Sigma^{*}\right)$.

We notice that $i\left(G_{1}\right)$ is the zero locus of a global section of $Q \otimes V_{2}^{*}$ in $G$. We claim that the restriction maps

$$
\begin{aligned}
r_{1}: H^{k(n-r)}\left(G, \Gamma^{a} \Sigma\right) \simeq \Gamma^{\psi(0, a)} V & \rightarrow H^{k(n-r)}\left(G_{1}, i^{*} \Gamma^{a} \Sigma\right), \\
r_{2}: H^{0}\left(G, \mathbf{S}^{d} \Sigma^{*}\right) & \rightarrow H^{0}\left(G_{1}, i^{*} \mathbf{S}^{d} \Sigma_{1}^{*}\right), \\
r_{3}: H^{0}\left(G, \Gamma^{\alpha} \Sigma\right) & \rightarrow H^{0}\left(G_{1}, i^{*} \Gamma^{\alpha} \Sigma\right)
\end{aligned}
$$

are surjective.

We prove that $r_{1}$ is surjective, and the others are easy. We use the Koszul resolution of the structure sheaf $\mathscr{O}_{i\left(G_{1}\right)}$ :

$$
0 \rightarrow \operatorname{det}\left(Q^{*} \otimes V_{2}\right) \rightarrow \cdots \rightarrow Q^{*} \otimes V_{2} \rightarrow \mathscr{O}_{G} \rightarrow \mathscr{O}_{i\left(G_{1}\right)} \rightarrow 0
$$

Therefore, we just need to prove that

$$
H^{k(n-r)+i}\left(G, \Gamma^{a} \Sigma \otimes \wedge^{i}\left(Q^{*} \otimes V_{2}\right)\right)=0
$$

for each $i \geq 1$. We then argue by contradiction. Assume that there exists a direct summand $\Gamma^{b} Q^{*}$ of $\wedge^{i}\left(Q^{*} \otimes V_{2}\right)$ such that $H^{k(n-r)+i}\left(G, \Gamma^{a} \Sigma \otimes \Gamma^{b} Q^{*}\right) \neq$ 
0 . We have $|b|=i$, and we may write $b=\left(b_{1}, \ldots, b_{n-r}\right) \geq 0$. Since $H^{k(n-r)}(G$, $\left.\Gamma^{a} \Sigma\right) \neq 0$, we have $a_{k} \geq n-r+k$ and $a_{k+1} \leq k$. Again by Bott's theorem, there exists a positive integer $t \leq n-r$ such that for each $1 \leq s \leq t$, there exists $i_{s} \geq 1$ with

$$
-b_{s}-(n-r+1-s)<a_{k+i_{s}}-\left(n-r+k+i_{s}\right),
$$

and $\sum_{s=1}^{t} i_{s}=i$. We then conclude that

$$
b_{s} \geq i_{s}+s+k-a_{k+i_{s}} \geq i_{s}+s \text {. }
$$

It contradicts the fact that $|b|=i$. Hence, we finish the proof of the claim.

We now consider on $G_{1}$ the multiplication

$$
\begin{aligned}
& m_{\bar{\alpha}, \widetilde{\alpha}}: H^{k(n-r)}\left(G_{1}, \Gamma^{\bar{a}} \Sigma_{1} \otimes \Gamma^{\widetilde{a}} V_{2}\right) \otimes H^{0}\left(G_{1}, \mathbf{S}^{d_{1}} \Sigma_{1}^{*} \otimes \mathbf{S}^{d_{2}} V_{2}^{*}\right) \\
& \quad \rightarrow H^{k(n-r)}\left(G_{1}, \Gamma^{\bar{\alpha}} \Sigma_{1} \otimes \Gamma^{\widetilde{\alpha}} V_{2}\right),
\end{aligned}
$$

where both sides are nonzero. Take the Serre duality; the multiplication

$$
\begin{aligned}
& H^{0}\left(G_{1}, \Gamma^{\bar{\alpha}} \Sigma_{1}^{*} \otimes K_{G_{1}} \otimes \Gamma^{\widetilde{\alpha}} V_{2}^{*}\right) \otimes H^{0}\left(G_{1}, \mathbf{S}^{d_{1}} \Sigma_{1}^{*} \otimes \mathbf{S}^{d_{2}} V_{2}^{*}\right) \\
& \rightarrow H^{0}\left(G_{1}, \Gamma^{\bar{a}} \Sigma_{1}^{*} \otimes K_{G_{1}} \otimes \Gamma^{\widetilde{a}} V_{2}^{*}\right)
\end{aligned}
$$

is surjective by statement (1) of this proposition. Therefore, the map $m_{\bar{\alpha}, \widetilde{\alpha}}$ is nontrivial.

We then consider the commutative diagram

$$
\begin{gathered}
H^{k(n-r)}\left(G, \Gamma^{a} \Sigma\right) \otimes H^{0}\left(G, \mathbf{S}^{d} \Sigma^{*}\right) \\
\downarrow r_{1} \otimes r_{2} \\
H^{k(n-r)}\left(G_{1}, i^{*} \Gamma^{a} \Sigma\right) \otimes H^{0}\left(G_{1}, i^{*} \mathbf{S}^{d} \Sigma^{*}\right) \\
\text { projection to direct summand } \downarrow \\
H^{k(n-r)}\left(G_{1}, \Gamma^{\bar{a}} \Sigma_{1} \otimes \Gamma^{\widetilde{a}} V_{2}\right) \otimes H^{0}\left(G_{1}, \mathbf{S}^{d_{1}} \Sigma_{1}^{*} \otimes \mathbf{S}^{d_{2}} V_{2}^{*}\right)
\end{gathered}
$$




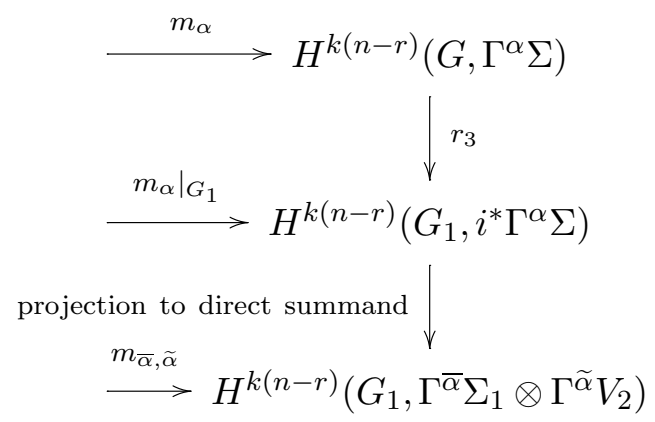

Since we know by (2) that all the vertical maps are surjective, and we have seen above that the map $m_{\bar{\alpha}, \widetilde{\alpha}}$ is nontrivial, we deduce that $m_{\alpha}$ is also nontrivial. Moreover, since $\Gamma^{\psi(0, \alpha)} V$ is an irreducible $\mathrm{GL}(V)$-module, $m_{\alpha}$ is surjective.

Since the multiplicity of each $\Gamma^{\alpha} \Sigma$ in $\Gamma^{a} \Sigma \otimes \mathbf{S}^{d} \Sigma^{*}$ is 1 and since $\Gamma^{\psi(0, \alpha)} V$ is an irreducible GL( $V)$-module, by Schur's lemma we deduce that the multiplication

$$
H^{k(n-r)}\left(G, \Gamma^{a} \Sigma\right) \otimes H^{0}\left(G, \mathbf{S}^{d} \Sigma^{*}\right) \rightarrow H^{k(n-r)}\left(G, \Gamma^{a} \Sigma \otimes \mathbf{S}^{d} \Sigma^{*}\right)
$$

is again surjective.

This concludes the proof.

Remark 1.5. Assume that we have $H^{i}\left(G, \Gamma^{\alpha} \Sigma\right) \neq 0, H^{j}\left(G, \Gamma^{\beta} \Sigma\right) \neq 0$, and $H^{i+j}\left(G, \Gamma^{\alpha} \Sigma \otimes \Gamma^{\beta} \Sigma\right) \neq 0$ for partitions $\alpha, \beta$ and integers $i, j>0$. The multiplication

$$
H^{i}\left(G, \Gamma^{\alpha} \Sigma\right) \otimes H^{j}\left(G, \Gamma^{\beta} \Sigma\right) \rightarrow H^{i+j}\left(G, \Gamma^{\alpha} \Sigma \otimes \Gamma^{\beta} \Sigma\right)
$$

is in general not surjective.

We may consider $G:=G(2,4)$. Let $\alpha=\beta=(3,1)$. Then $\Gamma^{(4,4)} \Sigma$ is a direct summand of $\Gamma^{(3,1)} \Sigma \otimes \Gamma^{(3,1)} \Sigma$. However, through restrictions on $G(2,3) \hookrightarrow$ $G$, we can see that the multiplication

$$
H^{2}\left(G, \Gamma^{(3,1)} \Sigma\right) \otimes H^{2}\left(G, \Gamma^{(3,1)} \Sigma\right) \rightarrow H^{4}\left(G, \Gamma^{(4,4)} \Sigma\right)
$$

is zero.

The cup products of line bundles on homogeneous varieties have been studied in $[\mathrm{DR}]$. It may be possible to prove Proposition 1.4 directly by applying the main theorem in $[\mathrm{DR}]$. 
$\S 2$. Proof of the main theorem when $\operatorname{dim}\left(F_{r}(X)\right)=2$

Under the assumptions of the main theorem, we will assume furthermore in this section that $\delta(n, \underline{d}, r)=2$. We notice that $\delta_{-}(n, \underline{d}, r) \geq 1$, and hence $F_{r}(X)$ is smooth and connected.

Proposition 2.1. Assume that $\delta(n, \underline{d}, r)=2$. Set $G:=G(r+1, V)$. Then, for any $1 \leq i \leq s$, the map

$$
H^{0}\left(F_{r}(X), K_{F_{r}(X)}\right) \otimes H^{0}\left(G, \mathbf{S}^{d_{i}} \Sigma^{*}\right) \rightarrow H^{0}\left(F_{r}(X), \mathbf{S}^{d_{i}} \Sigma^{*} \otimes K_{F_{r}(X)}\right)
$$

is surjective, except in the following cases:

- $X$ is a complete intersection of two quadrics in $\mathbf{P}^{5}$ and $r=1$;

- $X$ is a cubic in $\mathbf{P}^{4}$ and $r=1$.

Proof. Denote by $H$ the Plücker polarization on $G$. Set

$$
M=\left(\begin{array}{l}
\underline{d}+r \\
r+1
\end{array}\right)-n-1 .
$$

Then $K_{F_{r}(X)}=M H$. Since $\delta(n, \underline{d}, r)=2$, we can verify that $M \geq 0$ with equality only when $n=5, r=1$, and $\underline{d}=(2,2)$.

Let $W$ be the vector bundle

$$
\bigoplus_{i=1}^{s} \mathbf{S}^{d_{i}} \Sigma
$$

on $G$. We have the Koszul resolution of the structure sheaf $\mathscr{O}_{F_{r}(X)}$ :

$$
\left.0 \rightarrow \wedge^{\left(\frac{d}{r}+r\right.}\right) W \rightarrow \cdots \rightarrow \wedge^{n-1} W \rightarrow \cdots \rightarrow W \rightarrow \mathscr{O}_{G} \rightarrow \mathscr{O}_{F_{r}(X)} \rightarrow 0 .
$$

By splitting the Koszul resolution to short exact sequences, we see that in order to prove the proposition, we just need to prove that the multiplication

$$
\begin{aligned}
\Psi_{k, i} & : H^{k(n-r)}\left(G, \wedge^{k(n-r)} W \otimes \mathscr{O}_{G}(M H)\right) \otimes H^{0}\left(G, \mathbf{S}^{d_{i}} \Sigma^{*}\right) \\
& \rightarrow H^{k(n-r)}\left(G, \mathbf{S}^{d_{i}} \Sigma^{*} \otimes \wedge^{k(n-r)} W \otimes \mathscr{O}_{G}(M H)\right)
\end{aligned}
$$

is surjective for all $0 \leq k \leq r$ and $1 \leq i \leq s$.

We now assume that there exist $k$ and $i$ such that $\Psi_{k, i}$ is not surjective.

Considering the decomposition of the vector bundle $\wedge^{k(n-r)} W$ on $G$, we may write

$$
\wedge^{k(n-r)} W=\bigoplus_{\alpha} \Gamma^{\alpha} \Sigma .
$$


Then according to Proposition 1.4, we have $k \geq 1$, and there exists $\alpha$ such that

$$
\begin{aligned}
\alpha_{k} & \geq M+n-r+k, \\
\alpha_{k+1} & \geq M+k+1 .
\end{aligned}
$$

Moreover, we notice that $M+n-r+k=\left(\frac{d+r}{r+1}\right)-r-1+k$ and

$$
\operatorname{det} W=-\left(\begin{array}{l}
\underline{d}+r \\
r+1
\end{array}\right) H=\Gamma^{\left(\left(\frac{d}{r+1}+r\right), \ldots,\left(\frac{d}{r}+r\right)\right)} \Sigma .
$$

Hence, by Lemma 2.2 (take $s=r+1-k$ ), we have the following inequality:

$$
k(n-r) \geq\left[\left(\begin{array}{c}
r+\underline{d} \\
r
\end{array}\right)-\left(\begin{array}{c}
r-k+\underline{d} \\
r-k
\end{array}\right)\right]-k(r+1-k) .
$$

Since $\delta(n, \underline{d}, r)=2$, we conclude that

$$
\begin{gathered}
\frac{1}{r+1}\left(\begin{array}{c}
r+\underline{d} \\
r
\end{array}\right)+\frac{2}{r+1}+r+1-k \\
\geq \frac{1}{k}\left[\left(\begin{array}{c}
r+\underline{d} \\
r
\end{array}\right)-\left(\begin{array}{c}
r-k+\underline{d} \\
r-k
\end{array}\right)\right] .
\end{gathered}
$$

Since $k \leq r$, the function

$$
\frac{1}{k}\left[\left(\begin{array}{c}
r+\underline{d} \\
r
\end{array}\right)-\left(\begin{array}{c}
r-k+\underline{d} \\
r-k
\end{array}\right)\right]-\frac{1}{r+1}\left(\begin{array}{c}
r+\underline{d} \\
r
\end{array}\right)>0
$$

is a strictly increasing function of each $d_{i}$. By induction on $d_{i}$, we can check that the inequality (5) holds for some $k$ only in the following cases.

- $r=1$ :

(1.1) $\underline{d}=(2,2), M=0$, and $n=5$;

(1.2) $\underline{d}=(2,2,2,2), M=3$, and $n=8$;

(1.3) $\underline{d}=3, M=1$, and $n=4$;

(1.4) $\underline{d}=(3,3), M=5$, and $n=6$;

(1.5) $\underline{d}=(3,2,2), M=4$, and $n=7$;

(1.6) $\underline{d}=(4,2), M=6$, and $n=6$;

(1.7) $\underline{d}=5, M=9$, and $n=5$.

- $r=2$ :

(2.1) $\underline{d}=3, M=3$, and $n=6$; 
(2.2) $\underline{d}=(3,2), M=5$, and $n=8$.

- $r=3$ :

(3.1) $\underline{d}=(2,2,2), M=3$, and $n=11$.

In the next step, we will check in the above cases whether inequalities (3) and (4) actually hold for some $\alpha$. We will use the program LIE to get precise information on the decompositions of $\wedge^{k(n-r)} W$ and then show that these inequalities do not hold except in cases (1.1) and (1.3). This will conclude the proof of the proposition. Since this is only a computation, we just show the case $r=3$.

In case (3.1), we have $n=11, r=3$, and $M=3$.

When $k=1$, we have $\alpha_{1} \geq 12$ and $\alpha_{2} \geq 5$ by (3) and (4). However, the total weight for $\wedge^{8} W=\wedge^{8}\left(\mathbf{S}^{2} \Sigma \oplus \mathbf{S}^{2} \Sigma \oplus \mathbf{S}^{2} \Sigma\right)$ is only 16 .

When $k=3$, we have $\alpha_{1} \geq \alpha_{2} \geq \alpha_{3} \geq 14$ and $\alpha_{4} \geq 7$. But the total weight of $\wedge^{24}\left(\mathbf{S}^{2} \Sigma \oplus \mathbf{S}^{2} \Sigma \oplus \mathbf{S}^{2} \Sigma\right)$ is 48 . This is again impossible.

We have the decompositions

$$
\wedge^{t} \mathbf{S}^{2} \Sigma=\bigoplus_{i} \Gamma^{\alpha_{i}^{t} \Sigma}
$$

where

- $t=1, \alpha_{1}^{1}=(2,0,0,0)$;

- $t=2, \alpha_{1}^{2}=(3,1,0,0)$;

- $t=3, \alpha_{1}^{3}=(4,1,1,0)$, and $\alpha_{2}^{3}=(3,3,0,0)$;

- $t=4, \alpha_{1}^{4}=(4,3,1,0)$, and $\alpha_{2}^{4}=(5,1,1,1)$;

- $t=5, \alpha_{1}^{5}=(5,3,1,1)$, and $\alpha_{2}^{5}=(4,4,2,0)$;

- $t=6, \alpha_{1}^{6}=(5,4,2,1)$, and $\alpha_{2}^{6}=(4,4,4,0)$;

- $t=7, \alpha_{1}^{7}=(5,4,4,1)$, and $\alpha_{2}^{7}=(5,5,2,2)$;

- $t=8, \alpha_{1}^{8}=(5,5,4,2)$;

- $t=9, \alpha_{1}^{9}=(5,5,5,3)$;

- $t=10, \alpha_{1}^{10}=(5,5,5,5)$.

When $k=2$, we have $\alpha_{1} \geq \alpha_{2} \geq 13$ and $\alpha_{3} \geq 6$. Hence, $\Gamma^{(13,13,6,0)} \Sigma$ should be a subbundle of $\wedge^{16} W=\wedge^{16}\left(\mathbf{S}^{2} \Sigma \oplus \mathbf{S}^{2} \Sigma \oplus \mathbf{S}^{2} \Sigma\right)$. We have

$$
\wedge^{16}\left(\mathbf{S}^{2} \Sigma \oplus \mathbf{S}^{2} \Sigma \oplus \mathbf{S}^{2} \Sigma\right)=\bigoplus_{\substack{\left(s_{1}, s_{2}, s_{3}\right) \\ s_{1}+s_{2}+s_{3}=16}} \wedge^{s_{1}} \mathbf{S}^{2} \Sigma \otimes \wedge^{s_{1}} \mathbf{S}^{2} \Sigma \otimes \wedge^{s_{3}} \mathbf{S}^{2} \Sigma
$$

By the above list, we see that any $\alpha_{i}^{t}$ with $\left(\alpha_{i}^{t}\right)_{4}=0$ has $\left(\alpha_{i}^{t}\right)_{1} \leq 4$. Hence, we exclude this case by the Littlewood-Richardson rule.

We have thus excluded case (3.1). 
LemmA 2.2. Let $V$ be a vector space of dimension $r+1$, and let $\underline{d}=$ $\left(d_{1}, \ldots, d_{s}\right)$ be a sequence of integers at least 2 . Denote by $W$ the vector space $\mathbf{S}^{d_{1}} V \oplus \cdots \oplus \mathbf{S}^{d_{s}} V$. For any integer $s \geq 0$ and $1 \leq k \leq r$, we take an integer $t$ such that

$$
0<t<\left[\left(\begin{array}{c}
\underline{d}+r \\
r
\end{array}\right)-\left(\begin{array}{c}
\underline{d}+r-k \\
r-k
\end{array}\right)\right]-k s .
$$

Then for any irreducible component $\Gamma^{\lambda} V$ of $\wedge^{t} W$, we have

$$
\lambda_{k}<\left(\begin{array}{l}
\underline{d}+r \\
r+1
\end{array}\right)-s .
$$

The proof of this lemma is parallel to the proof of [DM, lemme 3.9]. For reader's convenience, we give the details.

Proof. For simplicity, we assume that $\underline{d}=d$. We denote by $X$ the Grassmannian parameterizing subspaces of dimension $r+1-k$ of $V$, and we denote by $Y$ the Grassmannian parameterizing subspaces of dimension $\left(\begin{array}{c}d+r-k \\ r-k\end{array}\right)$ of $W$. We denote by $\Sigma_{X}$ (resp., $\left.Q_{X}\right)$ and $\Sigma_{Y}$ (resp., $\left.Q_{Y}\right)$ the tautological subbundles (resp., quotient bundles) on $X$ and $Y$, respectively. There is a natural embedding $i: X \hookrightarrow Y$ so that $i^{*} \Sigma_{Y}=\mathbf{S}^{d} \Sigma_{X}$. Let $N$ be the normal bundle of $X \hookrightarrow Y$.

By Bott's theorem, we have $H^{0}\left(Y, \wedge{ }^{t} Q_{Y}\right)=\wedge^{t} W$. Considering the exact sequences

$$
\left.0 \rightarrow \mathscr{I}_{X} \otimes \wedge^{t} Q_{Y} \rightarrow \wedge^{t} Q_{Y} \rightarrow \wedge^{t} Q_{Y}\right|_{X} \rightarrow 0
$$

and, for each $l \geq 1$,

$$
\left.0 \rightarrow \mathscr{I}_{X}^{l+1} \otimes \wedge^{t} Q_{Y} \rightarrow \mathscr{I}_{X}^{l} \otimes \wedge^{t} Q_{Y} \rightarrow \wedge^{t} Q_{Y}\right|_{X} \otimes \mathbf{S}^{l} N^{*} \rightarrow 0,
$$

we see that there exists a filtration $\left(\Gamma_{l}\right)_{l \geq 0}$ on $\wedge{ }^{t} W$ so that

$$
\Gamma_{l} / \Gamma_{l+1} \hookrightarrow H^{0}\left(X, i^{*}\left(\wedge^{t} Q_{Y}\right) \otimes \mathbf{S}^{l} N^{*}\right)
$$

There is a filtration $\left(G_{m}\right)_{0 \leq m \leq d-1}$ of $i^{*} Q_{Y}$ such that

$$
G_{m} / G_{m+1}=\mathbf{S}^{d-m} Q_{X} \otimes \mathbf{S}^{m} \Sigma_{X}
$$

Denote by

$$
U=\operatorname{Gr}\left(i^{*} Q_{Y}\right)=\bigoplus_{m=0}^{d-1} \mathbf{S}^{d-m} Q_{X} \otimes \mathbf{S}^{m} \Sigma_{X}
$$


By the same argument in [DM, lemme 3.9], we have

$$
\Gamma_{l} / \Gamma_{l+1} \hookrightarrow H^{0}\left(X, i^{*}\left(\wedge^{t} Q_{Y}\right) \otimes \mathbf{S}^{l} N^{*}\right) \hookrightarrow H^{0}\left(X, \wedge^{t} U \otimes \mathbf{S}^{l} N^{*}\right)
$$

We now set $T=\left(\begin{array}{c}d+r \\ r\end{array}\right)-\left(\begin{array}{c}d+r-k \\ r-k\end{array}\right)$ as the rank of $U$. Then

$$
\wedge^{t} U=\operatorname{det} U \otimes \wedge^{T-t} U^{*}
$$

By definition of $t$, we have $T-t>k s$, and hence by considering the total weights, for any irreducible component $\Gamma^{\widehat{\alpha}} Q_{X}^{*} \otimes \Gamma^{\widehat{\beta}} \Sigma_{X}^{*}$ of $\wedge^{T-t} U^{*}$, we have $\widehat{\alpha}_{1}>s$. Moreover, we notice that

$$
\operatorname{det} U=\left(\operatorname{det} Q_{X}\right)^{\otimes\left(\begin{array}{l}
d+r \\
r+1
\end{array}\right)} \otimes\left(\operatorname{det} \Sigma_{X}\right)^{\otimes\left(\left(\begin{array}{l}
d+r \\
r+1
\end{array}\right)-\left(\begin{array}{l}
d+r-k \\
r-k+1
\end{array}\right)\right)} .
$$

Therefore, for any irreducible component $\Gamma^{\alpha} Q_{X} \otimes \Gamma^{\beta} \Sigma_{X}$ of $\wedge^{T-t} U$, we have

$$
\alpha_{k}<\left(\begin{array}{l}
d+r \\
r+1
\end{array}\right)-s .
$$

We notice that $N^{*}$ is a subbundle of $i^{*} \Omega_{Y}^{1}=\mathbf{S}^{d} \Sigma_{X} \otimes i^{*} Q_{Y}^{*}$. Hence, by the Littlewood-Richardson rule, for any irreducible component $\Gamma^{\alpha} Q_{X} \otimes \Gamma^{\beta} \Sigma_{X}$ of $\wedge^{T-t} U \otimes \mathbf{S}^{l} N^{*}$, we still have $\alpha_{k}<\left(\begin{array}{l}d+r \\ r+1\end{array}\right)-s$.

Therefore, by Bott's theorem, for any irreducible component $\Gamma^{\lambda} V$ of $\wedge^{t} W$, we have

$$
\lambda_{k}<\left(\begin{array}{l}
d+r \\
r+1
\end{array}\right)-s .
$$

LEMMA 2.3. If $\delta(n, \underline{d}, r)=2$, we have

$$
\operatorname{dim} H^{1}\left(F_{r}(X),\left.\Omega_{G}^{1}\right|_{F_{r}(X)}\right)=1,
$$

except when $X \subset \mathbf{P}^{5}$ is a smooth complete intersection of two quadrics and $r=1$.

Proof. We first notice that in the proof of [DM, théorème 3.4], the authors showed that

$$
\operatorname{dim} H^{1}\left(F_{r}(X),\left.\Omega_{G}^{1}\right|_{F_{r}(X)}\right)=1
$$

if $\delta_{-}(n, \underline{d}, r) \geq 2$. As we assume here that $\delta(n, \underline{d}, r)=2$, the cases when $\delta_{-}(n, \underline{d}, r)=1$ are

- $n=4, r=1, \underline{d}=3$; 
- $n=6, r=2, \underline{d}=3$;

- $n=5, r=1, \underline{d}=(2,2)$.

We then need to show that in the first two cases $H^{i}\left(G, \Omega_{G}^{1} \otimes \mathscr{I}_{F_{r}(X)}\right)=0$ for $i=1,2$. Again denote by $W$ the vector bundle $\bigoplus_{i} \mathbf{S}^{d_{i}} \Sigma$. By the Koszul resolution of $\mathscr{I}_{F_{r}(X)}$, we need to prove that

$$
H^{i+t-1}\left(G, \Omega_{G}^{1} \otimes \wedge^{t} W\right)=0
$$

for all $t \geq 1$ and $i=1,2$. We can use the program LIE to check the decompositions of $\wedge^{t} W$ in each case. By Bott's theorem, we conclude the proof of the lemma.

Theorem 2.4. Let $X$ be a very general complete intersection on $\mathbf{P}^{n}$ such that $\delta(n, \underline{d}, r)=2$. Then the Picard number $\rho\left(F_{r}(X)\right)$ is 1 .

Proof. By Lemma 1.1, Proposition 2.1, and Lemma 2.3, we have proved the theorem except when $r=1$ and $X$ is a complete intersection of two quadrics in $\mathbf{P}^{5}$ or when $r=1$ and $X$ is a cubic threefold. These two remaining cases were studied in Examples 0.4 and 0.5 .

\section{$\S 3$. Proof of the remaining cases}

In this section, we verify the remaining cases, namely, when $\operatorname{dim} F_{r}(X)=$ $\delta(n, \underline{d}, r) \geq 3$ and $1 \leq \delta_{-}(n, \underline{d}, r) \leq 2$ (see Theorem 0.2 ). The list is the following.

- $d_{1}=2$ :

(Q.1) $\underline{d}=(2)$, and $2 r+2 \leq n \leq 2 r+3$;

(Q.2) $\underline{d}=(2,2)$, and $2 r+3 \leq n \leq 2 r+4$;

(Q.3) $\underline{d}=(2,2,2), r=1, n=7$, and $\delta(n, \underline{d}, r)=3$;

(Q.4) $\underline{d}=(2,2,2), r=2, n=9$, and $\delta(n, \underline{d}, r)=3$;

- $d_{1}=3$ :

(C.1) $\underline{d}=(3), r=3, n=9$, and $\delta(n, \underline{d}, r)=4$;

(C.2) $\underline{d}=(3), r=2, n=7$, and $\delta(n, \underline{d}, r)=5$;

(C.3) $\underline{d}=(3), r=1, n=5$, and $\delta(n, \underline{d}, r)=4$;

(C.4) $\underline{d}=(3,2), r=1, n=6$, and $\delta(n, \underline{d}, r)=3$;

- $d_{1}=4$ :

(Qr) $\underline{d}=(4), r=1, n=5$, and $\delta(n, \underline{d}, r)=3$.

We will first study case by case the complete intersections of fewer than two quadrics and then discuss the others. 


\subsection{Case (Q.1)}

In case $(\mathrm{Q} .1), X \subset \mathbf{P}^{2 r+3}$ is a smooth quadric, and $F_{r+1}(X)$ has two isomorphic connected components, denoted by $S_{1}$ and $S_{2}$. Each $r$-plane in $X$ is contained in exactly one $(r+1)$-plane in each component of $F_{r+1}(X)$. Hence, $F_{r}(X) \simeq \mathbf{P}_{S_{1}}\left(\Sigma^{*}\right)$. In particular, $\rho\left(F_{r}(X)\right)=\rho\left(S_{1}\right)+1$. We then compute $\rho\left(F_{r}(X)\right)$ using the short exact sequence

$$
\left.0 \rightarrow \mathbf{S}^{2} \Sigma \rightarrow \Omega_{G}^{1}\right|_{F_{r}(X)} \rightarrow \Omega_{F_{r}(X)}^{1} \rightarrow 0 .
$$

We again have a resolution of $\mathscr{O}_{F_{r}(X)}$ :

$$
0 \rightarrow \wedge^{\frac{(r+1)(r+2)}{2}} \mathbf{S}^{2} \Sigma \rightarrow \cdots \rightarrow \wedge^{2} \mathbf{S}^{2} \Sigma \rightarrow \mathbf{S}^{2} \Sigma \rightarrow \mathscr{O}_{G} \rightarrow \mathscr{O}_{F_{r}(X)} \rightarrow 0 .
$$

By Bott's theorem, we have

$$
\begin{array}{r}
h^{j}\left(F_{r}(X), \mathbf{S}^{2} \Sigma\right)= \begin{cases}0 & \text { if } j \neq 2 \\
1 & \text { if } j=2\end{cases} \\
h^{j}\left(F_{r}(X),\left.\Omega_{G}^{1}\right|_{F_{r}(X)}\right)= \begin{cases}0 & \text { if } j \neq 1 \\
1 & \text { if } j=1 .\end{cases}
\end{array}
$$

Therefore, if $n=2 r+3, \rho\left(F_{r}(X)\right)=2$, and if $n=2 r+1$, the Picard number of each component of $F_{r}(X)$ is 1 .

If $n=2 r+2$, we can also compute that

$$
\begin{aligned}
& h^{j}\left(F_{r}(X), \mathbf{S}^{2} \Sigma\right)=0, \quad \text { for any } j ; \\
& h^{j}\left(F_{r}(X),\left.\Omega_{G}^{1}\right|_{F_{r}(X)}\right)= \begin{cases}0 & \text { if } j \neq 1 \\
1 & \text { if } j=1\end{cases}
\end{aligned}
$$

and hence, in this case $\rho\left(F_{r}(X)\right)=1$.

REMARK 3.1. A smooth quadric is a homogeneous variety. If $X$ is of dimension $2 r+2$ (resp., $2 r+1$ ), $X=G / P_{1}$ (resp., $G^{\prime} / P_{1}^{\prime}$ ), where $G$ (resp., $\left.G^{\prime}\right)$ is a complex simple Lie group of type $D_{r+2}$ (resp., $\left.B_{r+1}\right)$ and where $P_{1}$ (resp., $P_{1}^{\prime}$ ) is a maximal parabolic subgroup of $G$ (resp., $G^{\prime}$ ).

Hence, the above results can be easily deduced by [LM, Theorem 4.9]. Indeed, Landsberg and Manivel [LM] tell us much more: they imply that, if $\operatorname{dim} X=2 r+2$, then $F_{r}(X)$ is isomorphic to $G / P_{r+1, r+2}$ and $F_{r+1}(X)=$ $G / P_{r+1} \sqcup G / P_{r+2}$. Hence, $\rho\left(F_{r}(X)\right)=2$, and the Picard number of each component of $F_{r+1}(X)$ is 1 . Similarly, if $\operatorname{dim} X=2 r+1, F_{r}(X)=G^{\prime} / P_{r+1}^{\prime}$ and $\rho\left(F_{r}(X)\right)=1$. 


\subsection{Case (Q.2)}

In case (Q.2), we first consider $X \subset \mathbf{P}(V)=\mathbf{P}^{2 r+4}$, which is the smooth complete intersection of two quadrics. Then $F_{r}(X)$ is a Fano variety of dimension $2 r+2$.

Proposition 3.2. We have $\operatorname{dim} H^{1}\left(F_{r}(X), \Omega_{F_{r}(X)}^{1}\right)=2 r+6$. Hence, the Picard number $\rho\left(F_{r}(X)\right)$ is $2 r+6$.

The case when $r=1$ is already proved by Borcea [B2].

REMARK 3.3. It is relatively easy to prove that $\rho\left(F_{r}(X)\right) \geq 2 r+6$. Indeed,

$$
\operatorname{dim} H^{2 r+2}(X)_{\text {prim }}=\operatorname{dim} H^{r+1}\left(X, \Omega_{X}^{r+1}\right)_{\text {prim }}=2 r+5,
$$

and the Abel-Jacobi map $H^{2 r+2}(X)_{\text {prim }} \rightarrow H^{2}\left(F_{r}(X)\right)$ is injective.

Proof. We assume that $X$ is defined by two quadrics, $Q$ and $Q^{\prime}$.

We claim that

$$
\begin{aligned}
h^{j}\left(F_{r}(X), \mathbf{S}^{2} \Sigma\right) & = \begin{cases}0 & \text { if } j \neq 2,4, \\
2 r+5 & \text { if } j=2 ;\end{cases} \\
h^{j}\left(F_{r}(X),\left.\Omega_{G}^{1}\right|_{F_{r}(X)}\right) & = \begin{cases}1 & \text { if } j=1, \\
2 r+5 & \text { if } j=2, \\
0 & \text { if } j \neq 1,2,4 .\end{cases}
\end{aligned}
$$

From [W, Proposition 2.3.9], we know that there is a decomposition

$$
\wedge^{m} \mathbf{S}^{2} \Sigma=\bigoplus_{\lambda} \Gamma^{\lambda} \Sigma,
$$

where $|\lambda|=2 m$ and $\lambda \geq 0$ ranges over all partitions whose Frobenius notation has the form $\lambda=\left(\lambda_{1}-1, \ldots, \lambda_{t}-t \mid \lambda_{1}-2, \ldots, \lambda_{t}-t-1\right)$, where $t$ is the rank of $\lambda$.

We will compute

$$
H_{k, m}:=H^{k(r+4)}\left(G, \wedge^{m}\left(\mathbf{S}^{2} \Sigma \oplus \mathbf{S}^{2} \Sigma\right) \otimes \mathbf{S}^{2} \Sigma\right)
$$

to prove (6) using the Koszul resolution of $\mathscr{O}_{F_{r}(X)}$. More precisely, we will prove that only $H_{1, r+1}, H_{1, r+2}$, and $H_{2,2 r+4}$ may be nonzero and that the natural map $H_{1, r+2} \rightarrow H_{1, r+1}$ is surjective.

If $H_{k, m} \neq 0$, there exists a component $\Gamma^{a} \Sigma$ of

$$
\wedge^{m}\left(\mathbf{S}^{2} \Sigma \oplus \mathbf{S}^{2} \Sigma\right) \otimes \mathbf{S}^{2} \Sigma
$$


satisfying $a_{k} \geq r+4+k$ and $a_{k+1} \leq k$. We then assume that

$$
\Gamma^{a} \Sigma \subset \Gamma^{\beta} \Sigma \otimes \Gamma^{\gamma} \Sigma \otimes \mathbf{S}^{2} \Sigma,
$$

where

$$
\Gamma^{\beta} \Sigma \subset \wedge^{m_{1}} \mathbf{S}^{2} \Sigma
$$

and

$$
\Gamma^{\gamma} \Sigma \subset \wedge^{m_{2}} \mathbf{S}^{2} \Sigma
$$

for some $m_{1}+m_{2}=m$.

Since $a_{r+1} \leq \cdots \leq a_{k+1} \leq k$, we have by the Littlewood-Richardson rule that

$$
\sum_{i=k+1}^{r+1}\left(\beta_{i}+\gamma_{i}\right) \leq k(r+1-k)
$$

and both $\beta_{k+1}$ and $\gamma_{k+1}$ are at most $k$.

We denote by $p$ (resp., $q$ ) the largest integer such that $\beta_{p} \geq k+1$ (resp., $\left.\gamma_{q} \geq k+1\right)$. Clearly, $p, q \leq k$. Moreover, by (8), according to the definition of Frobenius notation, we have

$$
\sum_{i=1}^{p} \beta_{i}-(k+1) p \leq \sum_{i=k+1}^{r+1} \beta_{i}
$$

and

$$
\sum_{i=1}^{q} \gamma_{i}-(k+1) q \leq \sum_{i=k+1}^{r+1} \gamma_{i}
$$

On the other hand, since $a_{1} \geq \cdots \geq a_{k} \geq r+4+k$, we have, again by the Littlewood-Richardson rule, that

$$
\begin{aligned}
\sum_{i=1}^{p} \beta_{i}+\sum_{i=1}^{q} \gamma_{i}+k(k-p)+k(k-q) & \geq \sum_{i=1}^{k}\left(\beta_{i}+\gamma_{i}\right) \\
& \geq \sum_{i=1}^{k} a_{i}-2 \\
& \geq k(r+4+k)-2
\end{aligned}
$$

Combining all the above inequalities, we have

$$
k(r+3+k) \geq k(r+1+k)+p+q \geq k(r+4+k)-2 .
$$


Namely, if $H_{k, m} \neq 0$, we should have $k \leq 2$.

If $k=1$ and $H_{1, m} \neq 0$, both $\beta_{2}$ and $\gamma_{2}$ are at most 1 . Hence, there exists $1 \leq s \leq r+1$ so that $\beta_{1}=s+1$ and $\beta_{2}=\cdots=\beta_{s}=1>\beta_{s+1}=0$. Similarly, there exists $1 \leq t \leq r+1$ so that $\gamma_{1}=t+1$ and $\gamma_{2}=\cdots=\gamma_{t}=1>\gamma_{t+1}=0$. We then conclude that $r+1 \leq s+t \leq r+2$. Using Bott's theorem, a direct computation shows that

$$
H_{1, r+2} \simeq V^{\oplus(r+1)}
$$

and

$$
H_{1, r+1} \simeq\left(V^{*}\right)^{\oplus r},
$$

and the natural map in the Koszul complex induces a surjective map

$$
H_{1, r+2} \rightarrow H_{1, r+1} .
$$

If $k=2$ and $H_{2, m} \neq 0$, equality holds in (9) and (10). Therefore,

$$
m=m_{1}+m_{2}=\frac{1}{2}(|\beta|+|\gamma|)=2 r+4
$$

while $2(r+4)-2 r-4=4$.

Therefore, we have finished the proof of (6).

A similar analysis allows us to prove (7). Indeed, we can show that

$$
H_{t, m}^{\prime}:=H^{t}\left(G, \wedge^{m}\left(\mathbf{S}^{2} \Sigma \oplus \mathbf{S}^{2} \Sigma\right) \otimes \Omega_{G}^{1}\right)=0,
$$

unless $t=1$ and $m=0$, or $t=r+5$ and $r+2 \leq m \leq r+3$, or $t=2 r+9$ and $m=2 r+5$. Moreover, $\operatorname{dim} H_{1,0}^{\prime}=1$, and the natural map

$$
H_{r+5, r+3}^{\prime} \simeq V^{\oplus r} \rightarrow H_{r+5, r+2}^{\prime} \simeq\left(V^{*}\right)^{\oplus(r-1)}
$$

is surjective; this proves (7).

We have the following exact sequence:

$$
\left.0 \rightarrow \mathbf{S}^{2} \Sigma \oplus \mathbf{S}^{2} \Sigma \stackrel{\left(\partial Q, \partial Q^{\prime}\right)^{*}}{\longrightarrow} \Omega_{G}^{1}\right|_{F_{r}(X)} \rightarrow \Omega_{F_{r}(X)}^{1} \rightarrow 0 .
$$

Since $X$ is smooth, the map $\left(\partial Q, \partial Q^{\prime}\right)^{*}$ induces a surjective map between

$$
H^{2}\left(F_{r}(X), \mathbf{S}^{2} \Sigma \oplus \mathbf{S}^{2} \Sigma\right)=V^{\oplus 2} \rightarrow H^{2}\left(F_{r}(X),\left.\Omega_{G}^{1}\right|_{F_{r}(X)}\right)=V .
$$

We conclude that

$$
h^{1}\left(F_{r}(X), \Omega_{F_{r}(X)}^{1}\right)=2 r+6 .
$$

Since $F_{r}(X)$ is Fano, we have $h^{2,0}\left(F_{r}(X)\right)=0$, and this concludes the proof of the proposition.

Assume now that $X \subset \mathbf{P}^{2 r+3}$ is a very general complete intersection of two quadrics. We saw in Example 0.4 that $\rho\left(F_{r}(X)\right)=1$. 


\subsection{The remaining cases}

Some of the remaining cases are classical. For instance, in case (C.3), $X$ is a smooth cubic fourfold. By [BD], it is known that for $X$ very general, $F_{1}(X)$ is a very general deformation of $S^{[2]}$ for some polarized $K 3$ surface $S$; hence, $\rho\left(F_{1}(X)\right)=1$.

In the remaining cases $(\mathrm{Q} .3),(\mathrm{Q} .4),(\mathrm{C} .1)-(\mathrm{C} .4)$, and $(\mathrm{Qr})$, we have the following.

Proposition 3.4. Under the above assumptions, for each $d_{i}$, the multiplication

$$
\begin{aligned}
H^{\delta(n, \underline{d}, r)-2}\left(F_{1}(X), K_{F_{1}(X)}\right) \otimes H^{0} & \left(G, \mathbf{S}^{d_{i}} \Sigma^{*}\right) \\
& \rightarrow H^{\delta(n, \underline{d}, r)-2}\left(F_{1}(X), \mathbf{S}^{d_{i}} \Sigma^{*} \otimes K_{F_{1}(X)}\right)
\end{aligned}
$$

is surjective.

We omit the proof since it is again a direct application of Bott's theorem and Proposition 1.4. We also notice that in all the above cases, $\delta_{-}(n, \underline{d}, r)=$ 2. Therefore, by Debarre and Manivel's calculation (see [DM]),

$$
\operatorname{dim} H^{1}\left(F_{r}(X),\left.\Omega_{G}^{1}\right|_{F_{r}(X)}\right)=1 .
$$

Then by Lemma 1.1, we have completed the proof of the main theorem.

\section{$\S 4$. The cohomology class of varieties of planes of a cubic fivefold}

\subsection{An intersection formula}

In this section, we will always assume that $Z$ is a general smooth hypersurface of degree $d \geq 3$ in $\mathbf{P}(V)=\mathbf{P}^{n}$ and that the planes contained in $Z$ cover a divisor of $Z$; namely, we have $3 n-4-\left(\begin{array}{c}d+2 \\ 2\end{array}\right) \geq n-2$. The assumption holds when $Z$ is a cubic fivefold. We then automatically have $n-1 \geq d$; hence, the lines contained in $Z$ cover the whole variety.

We have the following correspondences:

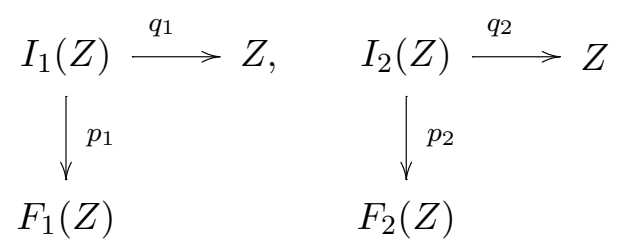


where $I_{1}(Z)$ and $I_{2}(Z)$ are the incidence varieties. Then $I_{1}(Z)=\mathbf{P}\left(\Sigma_{1}\right)$ and $I_{2}(Z)=\mathbf{P}\left(\Sigma_{2}\right)$, where $\Sigma_{1}$ and $\Sigma_{2}$ are, respectively, the tautological subbundle on $F_{1}(Z)$ and $F_{2}(Z)$. We denote respectively by $Q_{1}$ and $Q_{2}$ the tautological quotient bundle on $F_{1}(Z)$ and $F_{2}(Z)$, and we denote by $H_{1}$ and $H_{2}$ the respective Plücker polarization. We have $q_{1}^{*} \mathscr{O}_{Z}(1)=\mathscr{O}_{p_{1}}(1)$ and $q_{2}^{*} \mathscr{O}_{Z}(1)=\mathscr{O}_{p_{2}}(1)$, and then we set

$$
\begin{aligned}
& h_{1}=c_{1}\left(\mathscr{O}_{p_{1}}(1)\right), \quad h_{2}=c_{1}\left(\mathscr{O}_{p_{2}}(1)\right), \quad l=c_{1}\left(H_{1}\right), \quad l^{\prime}=c_{1}\left(H_{2}\right) ; \\
& c_{2}=c_{2}\left(\Sigma_{1}^{*}\right), \quad c_{2}^{\prime}=c_{2}\left(\Sigma_{2}^{*}\right), \quad c_{3}^{\prime}=c_{3}\left(\Sigma_{2}^{*}\right) .
\end{aligned}
$$

By definition, we have the following relations:

$$
\begin{aligned}
& h_{1}^{2}=h_{1} p_{1}^{*} l-p_{1}^{*} c_{2}, \\
& h_{2}^{3}=h_{2}^{2} p_{2}^{*} l^{\prime}-h_{2} p_{2}^{*} c_{2}^{\prime}+p_{2}^{*} c_{3}^{\prime} .
\end{aligned}
$$

For any $\alpha \in H^{n-1}(Z, \mathbf{Z})_{\text {prim }}$, we may write

$$
\begin{aligned}
& q_{1}^{*} \alpha=h_{1} p_{1}^{*} \alpha_{1}+p_{1}^{*} \alpha_{2}, \\
& q_{2}^{*} \alpha=h_{2}^{2} p_{2}^{*} \alpha_{1}^{\prime}+h_{2} p_{2}^{*} \alpha_{2}^{\prime}+p_{2}^{*} \alpha_{3}^{\prime},
\end{aligned}
$$

where $\alpha_{i} \in H^{*}\left(F_{1}(Z), \mathbf{Z}\right)$ and $\alpha_{i}^{\prime} \in H^{*}\left(F_{2}(Z), \mathbf{Z}\right)$. Denote

$$
\begin{aligned}
& \Phi(\alpha)=p_{1 *} q_{1}^{*} \alpha=\alpha_{1} \in H^{n-3}\left(F_{1}(Z), \mathbf{Z}\right), \\
& \Psi(\alpha)=p_{2 *} q_{2}^{*} \alpha=\alpha_{1}^{\prime} \in H^{n-5}\left(F_{2}(Z), \mathbf{Z}\right) .
\end{aligned}
$$

The following lemma is known (see $[\mathrm{BD}]$ ).

LEMma 4.1. For any $\alpha, \beta \in H^{n-1}(Z, \mathbf{Z})_{\text {prim }}$, we have

$$
\left(\Phi(\alpha) \cdot \Phi(\beta) \cdot l^{n-d}\right)_{F_{1}(Z)}=-d !(\alpha \cdot \beta)_{Z},
$$

and $c_{2} \cdot \alpha_{1}=0$.

We then consider the correspondence $C(Z)=\left\{([\Pi],[L]) \in F_{2}(Z) \times F_{1}(Z) \mid\right.$ $L \subset \Pi\}$ between $F_{1}(Z)$ and $F_{2}(Z)$ :

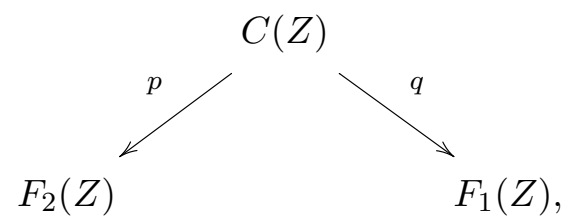


where $p$ and $q$ are the natural projections. Hence, $C(Z)=\mathbf{P}_{F_{2}(Z)}\left(\Sigma_{2}^{*}\right)$, and there is a tautological sequence of vector bundles on $C(Z)$ :

$$
0 \rightarrow q^{*} \Sigma_{1} \rightarrow p^{*} \Sigma_{2} \rightarrow \mathscr{O}_{p}(1) \rightarrow 0
$$

On the other hand, from the complex

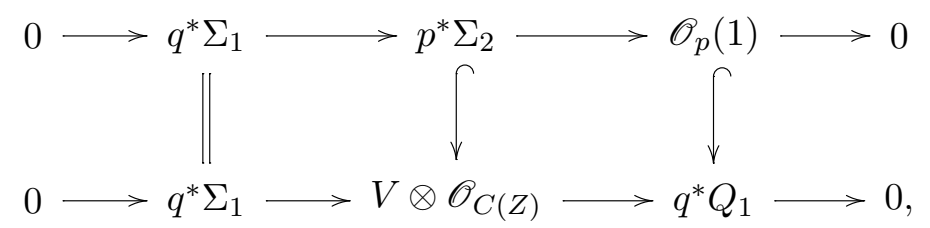

we see that $C(Z)$ is a natural subscheme of $\mathbf{P}_{F_{1}(Z)}\left(Q_{1}\right)$ :

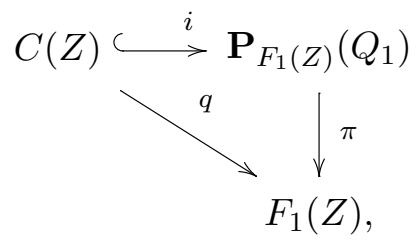

and $i^{*} \mathscr{O}_{\pi}(1)=\mathscr{O}_{p}(-1)$. Moreover, there is a natural short exact sequence on $\mathbf{P}_{F_{1}(Z)}\left(Q_{1}\right)$,

$$
0 \longrightarrow \pi^{*} \Sigma_{1} \longrightarrow \mathscr{K} \longrightarrow \mathscr{O}_{\pi}(-1) \longrightarrow 0
$$

where $i^{*} \mathscr{K}=p^{*} \Sigma_{2}$. Hence, we can describe $C(Z)$ to be the zero locus of a section of $S^{d-1} \mathscr{K}^{*} \otimes \mathscr{O}_{\pi}(1)$. Indeed, $F$ defines a section of $S^{d} \mathscr{K}^{*}$ which vanishes on $\pi^{*} S^{d} \Sigma_{1}^{*}$ and hence defines a section in

$$
H^{0}\left(\mathbf{P}_{F_{1}(Z)}\left(Q_{1}\right), S^{d-1} \mathscr{K}^{*} \otimes \mathscr{O}_{\pi}(1)\right),
$$

and $C(Z)$ is just the zero locus of this section.

Let $\eta=c_{1}\left(\mathscr{O}_{p}(1)\right)$. Since $C(Z)=\mathbf{P}_{F_{2}(Z)}\left(\Sigma_{2}^{*}\right)$ and by $(12)$, we have

$$
\begin{aligned}
& \eta^{3}=-\eta^{2} p^{*} l^{\prime}-\eta p^{*} c_{2}^{\prime}-p^{*} c_{3}^{\prime} \\
& q^{*} l=p^{*} l^{\prime}+\eta, \quad q^{*} c_{2}=p^{*} c_{2}^{\prime}+\eta q^{*} l=\eta^{2}+\eta p^{*} l^{\prime}+p^{*} c_{2}^{\prime},
\end{aligned}
$$

and for $\alpha_{1} \in H^{n-3}\left(F_{1}(Z), \mathbf{Z}\right)$ in $(11)$, we may write

$$
q^{*} \alpha_{1}=\eta^{2} p^{*} \alpha_{3}+\eta p^{*} \alpha_{4}+p^{*} \alpha_{5}
$$

By a direct computation, we have the following. 
Lemma 4.2. For any $\alpha \in H^{n-1}(X, \mathbf{Z})$, let $\alpha_{4}$ be as above. Then we have $\alpha_{4}=\Psi(\alpha)=p_{2 *} q_{2}^{*} \alpha$.

We now have the main result of this section.

Proposition 4.3. If $Z$ is a general cubic fivefold, for any $\alpha, \beta \in H^{5}(Z, \mathbf{Z})$, we have

$$
(\alpha \cdot \beta)_{Z}=\frac{1}{180}\left(\Psi(\alpha) \cdot \Psi(\beta) \cdot l^{\prime}\right)_{F_{2}(Z)} \cdot
$$

The main ingredient of the proof is the following calculation due to Voisin $([\mathrm{V} 2])$.

We first assume more generally that we are working on a hypersurface of degree $d$ in $\mathbf{P}^{n}$ with $3 n-4-\left(\begin{array}{c}d+2 \\ 2\end{array}\right)=n-2$.

Claim. For any $\alpha, \beta \in H^{n-1}(Z)_{\text {prim }}$, we define $\alpha_{1}, \beta_{1} \in H^{n-3}\left(F_{1}(Z)\right)$ as in (11). There exists a positive integer $N>0$ such that

$$
\left(q^{*} \alpha_{1} \cdot q^{*} \beta_{1} \cdot p^{*} l^{\prime}\right)_{C(Z)}=-N\left(\alpha_{1} \cdot \beta_{1} \cdot l^{n-d}\right)_{F_{1}(Z)} .
$$

We have already seen that $C(Z) \hookrightarrow \mathbf{P}_{F_{1}(Z)}\left(Q_{1}\right)$ is defined by a section of $S^{d-1} \mathscr{K}^{*} \otimes \mathscr{O}_{\pi}(1)$. We just need to calculate the cohomology class $q_{*}\left[p^{*} l^{\prime}\right]$ in $F_{1}(Z)$. By (13), this class is a polynomial of $l$ and $c_{2}$. By Lemma 4.1, $c_{2} \alpha_{1}=0$; hence, we are only interested in the coefficient of $l^{n-d}$. We may formally assume that $\Sigma_{1}^{*}=H_{1} \oplus \mathscr{O}_{F_{1}(Z)}$. Denote by $c_{1}\left(\mathscr{O}_{\pi}(1)\right)=\varepsilon$. We have seen that $i^{*} \varepsilon=-\eta$. As $S^{d-1} \mathscr{K}^{*} \otimes \mathscr{O}_{\pi}(1)$ is filtered with successive quotient

$$
\mathscr{O}_{\pi}(i) \otimes S^{d-i} \Sigma_{1}^{*}
$$

for $i=1, \ldots, d$, we have modulo $c_{2}$,

$$
\begin{aligned}
c_{\left(\begin{array}{c}
d+1 \\
2
\end{array}\right)}\left(S^{d-1} \mathscr{K}^{*} \otimes \mathscr{O}_{\pi}(1)\right) & =\prod_{1 \leq i \leq j \leq d}(i \varepsilon+(d-j) l) \\
& =d ! \varepsilon^{d} \prod_{1 \leq i \leq j \leq d-1}(i \varepsilon+(d-j) l) .
\end{aligned}
$$

By $(14), p^{*} l^{\prime}=q^{*} l-\eta=q^{*} l+\varepsilon$. Therefore,

$$
q_{*}[C(Z)]=\pi_{*}\left(d !\left(\varepsilon^{d} \prod_{1 \leq i \leq j \leq d-1}(i \varepsilon+(d-j) l)\right)\right) \bmod c_{2}
$$

and

$$
q_{*}\left[p^{*} l^{\prime}\right]=\pi_{*}\left(d !\left(\pi^{*} l+\varepsilon\right) \varepsilon^{d} \prod_{1 \leq i \leq j \leq d-1}(i \varepsilon+(d-j) l)\right) \bmod c_{2}
$$


We define the polynomial in two variables $M(x, y)=\prod_{1 \leq i \leq j \leq d-1}(i x+(d-$ j)y $=\sum_{i=1}^{\left(\begin{array}{l}d \\ 2\end{array}\right)} \alpha_{i} x^{i} y^{\left(\begin{array}{c}d \\ 2\end{array}\right)-i}$. By symmetry, we have $\alpha_{i}=\alpha_{\left(\begin{array}{c}d \\ 2\end{array}\right)-i}$ and it is easy to see that $\alpha_{i-1}<\alpha_{i}$ for $2 i \leq\left(\begin{array}{l}d \\ 2\end{array}\right)$.

On the other hand, $\pi_{*} \varepsilon^{n-2+i}=s_{i}\left(Q_{1}\right)=c_{i}\left(\Sigma_{1}\right)$; hence, we have $\pi_{*} \varepsilon^{n-2}=$ $1, \pi_{*} \varepsilon^{n-1}=-l, \pi_{*} \varepsilon^{n}=c_{2}$, and $\pi_{*} \varepsilon^{n-2+i}=0$, for $i \geq 3$. We conclude that

$$
q_{*}[C(Z)]=d !\left(\alpha_{n-2-d}-\alpha_{n-1-d}\right) l^{n-d-1} \bmod c_{2}
$$

and

$$
\begin{aligned}
q_{*}\left[p^{*} l^{\prime}\right] & =d !\left(\alpha_{n-2-d}-\alpha_{n-1-d}+\alpha_{n-3-d}-\alpha_{n-2-d}\right) l^{n-d} \\
& =d !\left(\alpha_{n-3-d}-\alpha_{n-1-d}\right) l^{n-d} \bmod c_{2} .
\end{aligned}
$$

Since we have $3 n-4-\left(\begin{array}{c}n+2 \\ 2\end{array}\right)=n-2$, then $(n-2-d)+(n-1-d)=\left(\begin{array}{c}d-1 \\ 2\end{array}\right)$, $d !\left(\alpha_{n-2-d}-\alpha_{n-1-d}\right)=0$, and $d !\left(\alpha_{n-3-d}-\alpha_{n-1-d}\right):=-N<0$. Hence, $q_{*}[C(Z)]=0 \bmod c_{2}$ and

$$
\left(q^{*} \alpha_{1} \cdot q^{*} \beta_{1} \cdot p^{*} l_{2}\right)_{C(Z)}=-N\left(\alpha_{1} \cdot \beta_{1} \cdot l^{n-d}\right)_{F_{1}(Z)} .
$$

Hence, we have proved Claim 1.

Proof of Proposition 4.3. We use the notation in the above calculation. Since

$$
q^{*} \alpha_{1}=\eta p^{*} \alpha_{4}+p^{*} \alpha_{5}, \quad q^{*} \beta_{1}=\eta p^{*} \beta_{4}+p^{*} \beta_{5} \in H^{3}(C(Z), \mathbf{Z}),
$$

by Lemma 4.2 , we have

$$
\left(q^{*} \alpha_{1} \cdot q^{*} \beta_{1} \cdot p^{*} l^{\prime}\right)_{C(Z)}=\left(\alpha_{4} \cdot \beta_{4} \cdot l^{\prime}\right)_{F_{2}(Z)}=\left(\Psi(\alpha) \cdot \Psi(\beta) \cdot l^{\prime}\right)_{F_{2}(Z)} .
$$

By Lemma 4.1 and Claim 1, we just need to show that $N=30$. In this case, $M(x, y)=(x+2 y)(2 x+2 y)(2 x+y)=2 x^{3}+7 x^{2} y+7 x y^{2}+2 y^{3}$. Therefore, $N=d !\left(\alpha_{n-d-1}-\alpha_{n-d-3}\right)=3 !(7-2)=30$.

\subsection{Proof of Theorem 0.8}

Let $U \subset \mathbf{P}\left(H^{0}(\mathbf{P}(V), \mathscr{O}(3))\right)$ be the open subset of a smooth cubic fivefold. There is the universal family of cubics $p r: \mathscr{Z} \rightarrow U$. We consider the monodromy action

$$
\rho: \pi_{1}(U, 0) \rightarrow \operatorname{Aut}\left(H^{5}(Z, \mathbf{Q}), \mathrm{I}\right)
$$


where I is the intersection form on $H^{5}(Z, \mathbf{Q})$. It is known that the Zariski closure of the image of $\rho$ is the full group $\operatorname{Sp}\left(H^{5}(Z, \mathbf{Q}), \mathrm{I}\right)$ (see, e.g., [PS]). For any $d$, we denote by $\operatorname{Hdg}^{2 d}(J Z):=H^{d, d}(J Z) \cap H^{2 d}(J Z, \mathbf{Q})$ the group of Hodge classes of $J Z$. As a corollary of the big monodromy group, we have the following.

LEMMA 4.4. We have $\operatorname{Hdg}^{2 d}(J Z)=\mathbf{Q}\left\langle\Theta^{d}\right\rangle$ for all $0 \leq d \leq 21$.

Proof. We consider the relative intermediate Jacobian $p r: \mathscr{J} \rightarrow U$. Then the Zariski closure of the monodromy action $\pi(U, 0) \rightarrow H^{1}(J Z, \mathbf{Q})$ is again the full symplectic group. The subspace of invariants in $\wedge^{2 d} H^{1}(J Z, \mathbf{Q})$, with respect to the full symplectic group, is 1-dimensional and is spanned by $\mathbf{Q}\left\langle\Theta^{d}\right\rangle$.

THEOREM 4.5. Let $Z$ be a general cubic fivefold. We consider the AbelJacobi map $\alpha: F_{2}(Z) \rightarrow J Z$. Then the cohomology class $\left[\alpha_{*}\left(F_{2}(Z)\right)\right]=$ $12\left[\Theta^{19} / 19 !\right]$.

Proof. We may assume that $Z$ is very general. By Theorem 0.3 in the introduction, we know that $\rho\left(F_{2}(Z)\right)=1$. Hence, we have $l^{\prime} \equiv x \alpha^{*} \Theta$ for some rational number $x$. By Lemma 4.4 , we may also write $\left[\alpha_{*}\left(F_{2}(Z)\right)\right]=$ $y\left[\Theta^{19} / 19\right.$ ! ] for some integer $y$. For any $\alpha, \beta \in H^{1}(J Z, \mathbf{Z})=H^{5}(Z, \mathbf{Z})$, we have

$$
\begin{aligned}
\left(\alpha \cdot \beta \cdot \frac{1}{20 !} \bigwedge^{20}[\Theta]\right)_{J Z} & =(\alpha \cdot \beta)_{Z} \\
& =\frac{1}{180}\left(\Psi(\alpha) \cdot \Psi(\beta) \cdot l^{\prime}\right)_{F_{2}(Z)} \\
& =\frac{1}{180} x y\left(\alpha \cdot \beta \cdot \frac{1}{19 !} \bigwedge^{20}[\Theta]\right)_{J Z}
\end{aligned}
$$

where the first equality holds by the definition of intermediate Jacobian, the second equality holds because of Proposition 4.3, and the last equality holds by projection formula. We have $x y=9$. On the other hand, we know from [IM, Corollary 10, Remark] that $\left(l^{\prime 2}\right)_{F_{2}(X)}=2835$. Hence, $x^{2} y=$ $2835 /(21 \cdot 20)=27 / 4$. We deduce that $x=3 / 4$ and $y=12$.

REMARK 4.6. It is not difficult to prove that for any smooth cubic fivefold $Z$, the variety of plane $F_{2}(Z)$ is always of dimension 2. Moreover, if $F_{2}(Z)$ is smooth, the Abel-Jacobi map $\alpha$ is generically injective. Hence, $J Z$ has a subvariety of dimension 2 whose cohomology class is $12\left[\Theta^{19} / 19\right.$ !]. 
Acknowledgments. Thanks are owed to Olivier Debarre, Laurent Manivel, and Claire Voisin for helpful discussions, to Frédéric Han for help with the program LIE, and to Ciprian Borcea and Xavier Roulleau for their remarks.

\section{REFERENCES}

[AK] A. B. Altman and S. L. Kleiman, Foundations of the theory of Fano schemes, Compos. Math. 34 (1977), 3-47.

[BVV] W. Barth and A. Van de Ven, Fano varieties of lines on hypersurfaces, Arch. Math. (Basel) 31 (1978), 96-104.

[BD] A. Beauville and R. Donagi, La variété des droites d'une hypersurface cubique de dimension 4, C. R. Math. Acad. Sci. Paris 301 (1985), 703-706.

[BM] S. Bloch and J. P. Murre, On the Chow groups of certain types of Fano threefolds, Compos. Math. 39 (1979), 47-105.

[BV] L. Bonavero and C. Voisin, Fano schemes and Moishezon manifolds (in French), C. R. Math. Acad. Sci. Paris 323 (1996), 1019-1024.

[B1] C. Borcea, Deforming varieties of $k$-planes of projective complete intersections, Pacific J. Math. 143 (1990), 25-36.

[B2] - "Homogeneous vector bundles and families of Calabi-Yau threefolds, II" in Several Complex Variables and Complex Geometry (Santa Cruz, 1989), Proc. Sympos. Pure Math. 52, Part 2, Amer. Math. Soc., Providence, 1991, 83-91.

[Bot] R. Bott, Homogeneous vector bundles, Ann. of Math. (2) 66 (1975), 203-248.

[CG] C. H. Clemens and P. A. Griffiths, The intermediate Jacobian of the cubic threefold, Ann. of Math. (2) 95 (1972), 281-356.

[DM] O. Debarre and L. Manivel, Sur la variété des espaces linéaires contenus dans une intersection complète, Math. Ann. 312 (1998), 549-574.

[DR] I. Dimitrov and M. Roth, Cup products of line bundles on homogeneous varieties and generalized PRV components of multiplicity one, preprint, arXiv:0909.2280v1 [math.AG]

[ELV] H. Esnault, M. Levine, and E. Viehweg, Chow groups of projective varieties of very small degree, Duke Math. J. 87 (1997), 29-58.

[IM] A. Iliev and L. Manivel, Cubic hypersurfaces and integrable systems, Amer. J. Math. 130 (2008), 1445-1475.

$[\mathrm{J}] \quad$ Z. Jiang, On the restriction of holomorphic forms, Manuscripta Math. 124 (2007), 173-182.

[LM] J. M. Landsberg and L. Manivel, On the projective geometry of rational homogeneous varieties, Comment. Math. Helv. 78 (2003), 65-100.

[PS] C. Peters and J. Steenbrink, Mixed Hodge Structures, Ergeb. Math. Grenzgeb. (3) 52, Springer, Berlin, 2008.

[P] G. P. Pirola, Base number theorem for abelian varieties: An infinitesimal approach, Math. Ann. 282 (1988), 361-368.

[Re] M. Reid, The complete intersection of two or more quadrics, Ph.D. dissertation, University of Cambridge, Cambridge, England, 1972.

[Ro] X. Roulleau, Elliptic curve configurations on Fano surfaces, Manuscripta Math. 129 (2009), 381-399.

[S] J. Spandaw, Noether-Lefschetz Problems for Degeneracy Loci, Mem. Amer. Math. Soc. 161 (2003), no. 764. 
[W] J. Weyman, Cohomology of Vector Bundles and Syzygies, Cambridge Tracts in Math. 149, Cambridge University Press, Cambridge, 2003.

[V1] C. Voisin, Hodge Theory and Complex Algebraic Geometry, II, Cambridge Stud. Adv. Math. 77, Cambridge University Press, Cambridge, 2003.

[V2] - Coniveau 2 complete intersections and effective cones, Geom. Funct. Anal. 19 (2010), 1494-1513.

Mathématiques Bâtiment 425

Université Paris-Sud

F-91405 Orsay

France

zhi.jiang@math.u-psud.fr 